\title{
Análise das hospitalizações por doenças cardiovasculares associadas a fatores ambientais
}

Variáveis climáticas como a precipitação, umidade relativa e temperatura, podem afetar a saúde humana de forma direta e indireta devido ao fato do corpo humano estar em permanente contato com o meio ambiente atmosférico. As doenças do aparelho circulatório (DAC) representam um importante problema de saúde pública no Brasil, por serem a principal causa de mortalidade, sendo também uma das principais causas de hospitalização. Diante desse contexto, este estudo tem como objetivo analisar o grau de relação entre hospitalizações por DAC e fatores climáticos no município de Campina Grande-PB. O atual estudo tratase de uma pesquisa ecológica de séries temporais para o período compreendido entre 1998 e 2013. Inicialmente foram analisadas as internações por DAC, em seguida, essas internações foram associadas a valores médios das variáveis climáticas medidas no município. Os métodos estatísticos utilizados foram o teste de Shapiro-Wilk, a correlação de Pearson e regressão linear múltipla. Esse estudo encontrou correlação entre os elementos climáticos e internações por doenças hipertensivas. A correlação verificada com os elementos climáticos indica que as internações por DAC apresentam correlações de moderadas a fortes. As variáveis temperatura média e umidade relativa do ar são as que mais influenciam no comportamento destas doenças. Os elementos climáticos juntos explicam $43 \%$ das internações por doenças hipertensivas, $36 \%$ das internações por doenças isquêmicas do coração e $34 \%$ das internações por insuficiência cardíaca.

Palavras-chave: Variáveis climáticas; Doenças do sistema circulatório; Regressão linear múltipla.

\section{Analysis of hospitalizations for cardiovascular diseases associated with climate factors}

\begin{abstract}
Climatic variables such as precipitation, relative humidity and temperature, can affect human health directly and indirectly because the human body is in permanent contact with the atmospheric environment. Diseases of the circulatory system (DCS) represent an important public health problem in Brazil, as they are the main cause of mortality and are a major cause of hospitalization. Given this context, this study aims to analyse the degree of relationship between hospitalizations for DCS and climatic factors in the city of Campina Grande-PB. The current study is an ecological survey of time series for the period between 1998 and 2013 . Initially, hospitalizations for DCSwere analyzed, and then these hospitalizations were associated with average values of the climatic variables measured in the municipality. The statistical methods used were the Shapiro-Wilk test, Pearson's correlation and multiple linear regression. This study found a correlation between climatic elements and hospitalizations for hypertensive diseases. The correlation verified with the climatic elements indicates that the hospitalizations due to DCS present moderate to strong correlations. The variables average temperature and relative humidity are the ones that most influence the behaviour of these diseases. The climatic elements together explain $43 \%$ of hospitalizations for hypertensive diseases, $36 \%$ of hospitalizations for ischemic heart diseases and $34 \%$ of hospitalizations for heart failure.
\end{abstract}

Keywords: Climatic variables; Diseases of the circulatory system; Multiple linear regression.

Topic: Meteorologia, Climatologia e Mudanças Climáticas

Reviewed anonymously in the process of blind peer.
Received: 04/03/2021

Approved: 23/03/2021
Juliana Meira de Vasconcelos Xavier (iD) Universidade Federal de Campina Grande, Brasil http://lattes.cnpq.br/2221607460684235 http://orcid.org/0000-0003-0603-1943 juliana-mvasconcelos@hotmail.com

Luana Andrade Lima Querino (iD

Universidade Federal de Campina Grande, Brasil http://lattes.cnpq.br/3979403454435718 http://orcid.org/0000-0001-5874-6006

luanaandradelima@gmail.com

\section{Pedro Vieira de Azevedo}

Universidade Federal de Campina Grande, Brasil http://lattes.cnpq.br/0324478957720406 http://orcid.org/0000-0002-3136-5219

juliana-mvasconcelos@hotmail.com

DOI: 10.6008/CBPC2179-6858.2021.003.0015

\author{
Fabrício Daniel Santos Silva (iD) \\ Universidade Federal de Alagoas, Brasil \\ http://lattes.cnpq.br/9324908747974694 \\ http://orcid.org/0000-0002-3185-6413 \\ juliana-mvasconcelos@hotmail.com \\ Mara Karinne Lopes Veriato Barros (i) \\ Universidade Federal de Campina Grande, Brasil \\ http://lattes.cnpq.br/9198696436575198 \\ http://orcid.org/0000-0003-1215-9599 \\ karinnelv@yahoo.com.br \\ Helder Morais Mendes Barros (iD) \\ Universidade Federal de Campina Grande, Brasil \\ http://lattes.cnpq.br/7955183658651586 \\ http://orcid.org/0000-0001-8950-3023 \\ hmmbbr@yahoo.com.br
}

Referencing this:

XAVIER, J. M. V.; QUERINO, L. A. L.; AZEVEDO, P. V.; SILVA, F. D. S.; BARROS, M. K. L. V.; BARROS, H. M. M.. Análise das hospitalizações por doenças cardiovasculares associadas a fatores ambientais. Revista Ibero Americana de Ciências Ambientais, v.12, n.3, p.155-168, 2021. DOI: http://doi.org/10.6008/CBPC2179-6858.2021.003.0015 


\section{INTRODUÇÃO}

Existe uma preocupação crescente em entender o impacto das variáveis meteorológicas na epidemiologia das doenças. Os fatores ambientais atuam sobre a dinâmica da ocorrência de várias morbidades, em especial das DAC, aumentando as taxas de internações e intensificação da doença.

Extremos climáticos afetam diretamente a saúde humana. Temperaturas baixas podem causar queda na imunidade do organismo, além de agravar males como artrites, sinusites. As altas temperaturas por sua vez, provocam a incidência de choques térmicos, exaustão e cãibras pelo calor. As doenças circulatórias também são influenciadas pelos elementos climáticos, dentre eles, as amplitudes térmicas, que contribuem para a vasodilatação e vasoconstrição do sistema circulatório.

Atualmente diversas sintomatologias como, por exemplo, dores de cabeça, nervosismo, cansaço e perturbações circulatórias podem ser atribuídas às condições meteorológicas. Frequentemente atribui-se ao tempo e ao clima a responsabilidade por diversas enfermidades (MURARA, 2012). Entretanto os elementos climáticos não são fatores determinantes dessas enfermidades.

Diante disso, os parâmetros climáticos como: precipitação, umidade, temperatura, pressão atmosférica, insolação e velocidade do vento, podem afetar a saúde humana de forma direta (sensação de conforto, morbidade por doenças sistêmicas, mortalidade) e indireta (doenças infecciosas transportadas por vetores), devido ao fato do corpo humano está em permanente contato com o meio ambiente atmosférico pelo intermédio de trocas térmicas hídricas e gasosas. As mudanças nos parâmetros de variáveis meteorológicas ocasionam variações fisiológicas consideráveis, agravando algumas doenças pré-existentes e favorecendo o aparecimento de outras, tornando-se um problema de saúde pública (PITTON et al., 2004; SILVA et al., 2013).

Nesse contexto, considerando a grande sensibilidade dos pacientes cardíacos ao estresse térmico ambiental, é possível supor que esses pacientes têm um mecanismo de termo regulação hipotalâmico pobre. Tanto exposição a baixas e altas temperaturas associado com baixa e alta umidade, podem causar forte pressão sobre pacientes cardíacos (GONÇALVES et al., 2007).

Keatinge et al. (1984) afirmam que as condições de exposição a temperaturas baixas ocasionam efeitos diferentes mediados pela ativação tanto da atividade do sistema nervoso simpático quanto do sistema coagulação. A vasoconstrição induzida pelas baixas temperaturas provoca um significativo aumento da carga de trabalho do coração.

Conforme Serrano Junior et al. (2009) as doenças do aparelho circulatório, designadas pelos códigos CID 100 a 199, representam as doenças que acometem o coração, a circulação pulmonar, as cerebrovasculares, as artérias, as veias e os vasos linfáticos. As doenças do aparelho cardiovascular (DAC) representam, atualmente, uma das principais causas de morbidade e a principal causa de mortalidade. As doenças hipertensivas, doenças isquêmicas e insuficiência cardíaca, representam as principais causas de hospitalizações no município de Campina Grande.

É de grande relevância estudos que correlacionem o clima e saúde, pois identificando a possível 
influência climática na ocorrência de morbidades, poderão ser traçadas medidas profiláticas que minimizem a intensidade da ocorrência de adoecimento por determinado tipo de doença.

A atual pesquisa foi desenvolvida visando alcançar o seguinte objetivo: Analisar as hospitalizações por doenças cardiovasculares associadas a fatores climáticos no município de Campina Grande-PB.

\section{MATERIAIS E MÉTODOS}

Esta pesquisa foi realizada com dados relativos ao município de Campina Grande, localizado no Estado da Paraíba (PB), do período de 1998 a 2013. Trata-se de um estudo ecológico do tipo investigação agregado-observacional, de referência temporal-longitudinal, visto que uma mesma área é investigada em momentos distintos. 0 município de Campina Grande está a uma altitude média de 555 metros acima do nível do mar, com $7^{\circ} 13^{\prime} 11^{\prime \prime}$ de latitude Sul e $35^{\circ} 52^{\prime} 31^{\prime \prime}$ de longitude Oeste, distante a $120 \mathrm{~km}$ da Capital do Estado, João Pessoa. A área do município de Campina Grande abrange 593,026 Km², contando com uma população estimada em 385.213habitantes, segundo dados do Instituto Brasileiro de Geografia e Estatística.

Está inserido na unidade geoambiental do Planalto da Borborema e incluído na área geográfica de abrangência do semiárido brasileiro. Apesar disso, por estar acima de quinhentos metros de altitude, possui um clima com temperaturas mais moderadas, considerado Tropical com estação seca (As, de acordo com a classificação climática de Köppen-Geiger). A estação chuvosa se inicia em maio com término em setembro, podendo se estender até outubro.

Os dados sobre as internações hospitalares foram buscados no Sistema de Informações Hospitalares do SUS (SIH/SUS), gerenciados pelo Ministério da Saúde, por meio da Secretaria de Assistência à Saúde, em conjunto com as secretarias de Saúde do Estado da Paraíba e Secretaria Municipal de Saúde do município de Campina Grande, ou seja, as unidades hospitalares, participantes do SUS (públicas ou particulares conveniadas), enviam as informações das internações efetuadas pela AlH - Autorização de Internações Hospitalares, para os gestores Estaduais e Municipais esses dados são processados e disponibilizados pelo DATASUS. O período de análise dessa pesquisa foi de janeiro de 1998 a dezembro de 2013.

Os dados meteorológicos foram coletados na estação climatológica principal do Instituto Nacional de Meteorologia (INMET) localizado na Empresa Brasileira de Pesquisa Agropecuária (EMBRAPA) CNPA (Centro Nacional de Pesquisa Algodoeira). Foram considerados os dados de umidade relativa do ar, temperatura do ar máxima, média e mínima, precipitação pluviométrica e pressão atmosférica com base nas médias mensais para a série histórica de 1998 a 2013.

\section{Análise Estatística dos Dados}

A partir dos dados meteorológicos e da saúde iniciou-se a análise estatística. Foram utilizados os totais mensais dos registros de precipitação pluviométrica, e as médias mensais da temperatura, umidade relativa do ar e pressão atmosférica.

Para a escolha das enfermidades dentro do grupo de doenças do aparelho circulatório, foi considerada a alta prevalência de internações hospitalares pelas doenças que compõem o grupo das doenças 
do aparelho circulatório no município referido. Os dados filtrados pelo DATASUS são relativos apenas aos residentes de Campina Grande, já que os hospitais do município atendem também populações de outras cidades. Foram selecionados os códigos que abrangem o mesmo grupo de causa de acordo com o CID 10, ou seja, cada enfermidade recebe um código, e se optou trabalhar com o universo que a mesma abrange, por exemplo, para doenças isquêmicas do coração os códigos referentes são 120 a I25, de modo que I20 (angina pectoris), 121 (infarto agudo do miocárdio), 122 (infarto do miocárdio recorrente), e assim por diante, esses, portanto representam o grupo das doenças isquêmicas do coração, ou seja, um subgrupo que faz parte de um grupo maior das DAC.

Em relação a análise dos dados a correlação de Pearson foi utilizada a fim de identificar o grau de associação entre as variáveis climáticas (temperatura do ar, precipitação pluviométrica e umidade relativa do ar e pressão atmosférica) e o número de casos anuais. Este coeficiente normalmente representado pela letra $r$ é obtido a partir da seguinte equação:

$$
r=\frac{\sum(x-\bar{x})(y-\bar{y})}{\sqrt{\sum(x-\bar{x})^{2}} \sum(y-\bar{y})^{2}}
$$

Onde: $\mathrm{r}$ varia entre -1 e 1 ; $x$ e y são as variáveis independente e dependente e suas médias, respectivamente. Os dados foram analisados através da regressão linear múltipla que é uma técnica estatística para modelar e investigar a relação entre variáveis. A regressão linear múltipla é uma variação mais geral e comum da regressão linear simples. É um conjunto de técnicas estatísticas que possibilitam a avaliação de uma variável dependente (preditando) com diversas variáveis independentes (preditores). 0 resultado é uma equação linear que pode ser utilizada para estimar o preditando como uma combinação linear dos preditores.

O modelo de regressão linear múltipla é dado pela seguinte equação.

$$
y_{i}=\beta_{0}+\beta_{1} x_{1}+\beta_{2} x_{2}+\ldots+\beta_{k} x_{k}+\varepsilon
$$

Onde: $Y=$ Variável dependente (preditando); $\mathrm{X}=$ Variável independente (preditor).

Em que $\beta_{0}, \beta_{1}, \beta_{2}, \ldots, \beta_{k}$ são parâmetros a serem estimados com os dados e $\varepsilon$ representa o erro aleatório. Na prática as estimativas desses parâmetros são obtidas a partir de uma amostra de $n$ observações como apresentado na

$$
\hat{y}=\hat{\beta}_{0}+\hat{\beta}_{1} x_{1}+\hat{\beta}_{2} x_{2}+\ldots+\hat{\beta}_{k} x_{k}
$$
equação abaixo.

Os coeficientes de determinação da regressão linear múltipla $\left(r^{2}\right)$ e o coeficiente de determinação ajustado $\left(r^{2}\right)$ são encontrados de forma semelhante à da regressão linear simples.

\section{RESULTADOS E DISCUSSÃO}

\section{Avaliação das correlações entre Clima e Doenças}

Inicialmente foram determinadas as correlações de Pearson $(r)$ entre os totais mensais no período dos anos de janeiro de 1998 a dezembro de 2013, dos números de internações dos grupos das DAC: Doenças Hipertensivas (DH), Doenças Isquêmicas do Coração (DISC) e Insuficiência Cardíaca (IC), com a média mensal das variáveis meteorológicas: Temperatura média $\left(T_{\text {med }}\right)$, Temperatura máxima $\left(T_{\text {max }}\right)$, Temperatura mínima 
$\left(T_{\min }\right)$; Umidade relativa do ar (UR); Precipitação pluvial (Pp) e Pressão atmosférica(P).

\section{Correlações do Grupo das Doenças Hipertensivas (DH) e a Variáveis Meteorológicas}

De acordo com os resultados de $r$, verificou-se que as temperaturas (média, máxima e mínima) apresentaram no período de janeiro de 1998 a dezembro de 2013 uma predominância nos valores das correlações positivas, apresentando correlações médias, na faixa de 0,30 a 0,60. Essa correlação positiva indica que à medida que os valores das temperaturas (média, máxima e mínima) aumentam, há uma tendência dos valores dos números das internações das doenças hipertensivas também crescer. Resultado semelhante foi encontrado no estudo de Schwartz et al. (2004), no qual observou-se que as internações hospitalares por todas as doenças cardíacas aumentaram com a temperatura média no mesmo dia. As Figuras 1, 2 e 3 ilustram as correlações lineares através dos diagramas de dispersões. Através de suas variáveis apresentam uma correlação linear quando os pontos do diagrama de dispersões se aproximam de uma reta.

Tabela 1: Matriz de Correlação DH versus Variáveis Meteorológicas.

\begin{tabular}{lllllll}
\hline Ano & $\mathbf{T}_{\text {med }}$ & $\mathbf{T}_{\max }$ & $\mathbf{T}_{\min }$ & UR & Pp & $\mathbf{P}$ \\
\hline 1998 & $-0,14$ & $-0,23$ & $-0,12$ & 0,09 & 0,43 & 0,27 \\
1999 & 0,59 & 0,66 & 0,55 & $-0,51$ & $-0,47$ & $-0,57$ \\
2000 & 0,34 & 0,39 & $-0,01$ & $-0,56$ & $-0,57$ & $-0,19$ \\
2001 & 0,19 & $-0,14$ & 0,55 & 0,29 & 0,31 & $-0,69$ \\
2002 & $-0,08$ & 0,19 & $-0,24$ & $-0,48$ & $-0,38$ & 0,17 \\
2003 & 0,38 & 0,63 & 0,15 & $-0,77$ & $-0,80$ & $-0,12$ \\
2004 & $-0,07$ & $-0,07$ & 0,03 & 0,17 & 0,59 & $-0,06$ \\
2005 & 0,61 & 0,57 & 0,58 & $-0,17$ & $-0,29$ & $-0,53$ \\
2006 & 0,01 & $-0,13$ & 0,16 & 0,15 & 0,38 & $-0,09$ \\
2007 & 0,46 & 0,47 & 0,44 & $-0,53$ & $-0,07$ & $-0,35$ \\
2008 & 0,16 & 0,33 & 0,03 & $-0,63$ & $-0,20$ & 0,01 \\
2009 & 0,22 & 0,12 & 0,30 & $-0,02$ & 0,19 & $-0,29$ \\
2010 & $-0,13$ & $-0,15$ & $-0,14$ & $-0,06$ & $-0,17$ & 0,00 \\
2011 & $-0,14$ & 0,02 & $-0,27$ & $-0,16$ & $-0,25$ & 0,20 \\
2012 & $-0,06$ & $-0,21$ & 0,11 & 0,47 & 0,56 & 0,07 \\
2013 & 0,62 & 0,51 & 0,66 & $-0,37$ & $-0,02$ & $-0,60$ \\
\hline
\end{tabular}

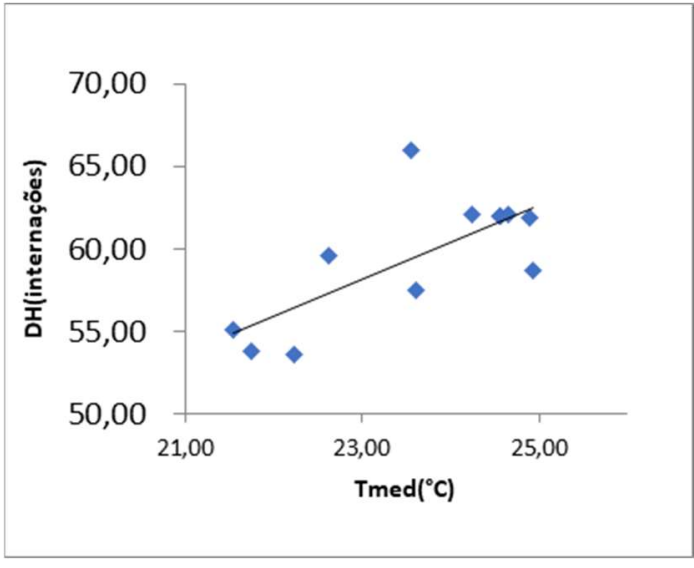

Figura 1: Diagrama de Dispersão Doenças Hipertensivas versus Tmed.

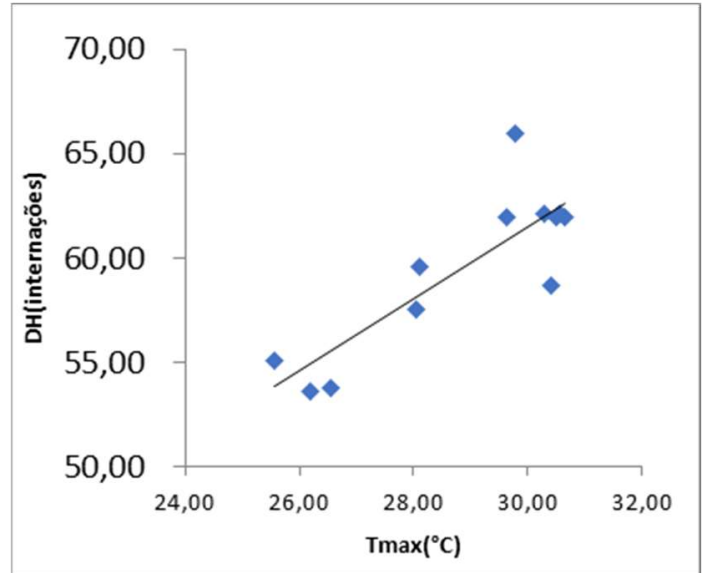

Figura 2: Diagrama de Dispersão Doenças Hipertensivas versus Tmax. 


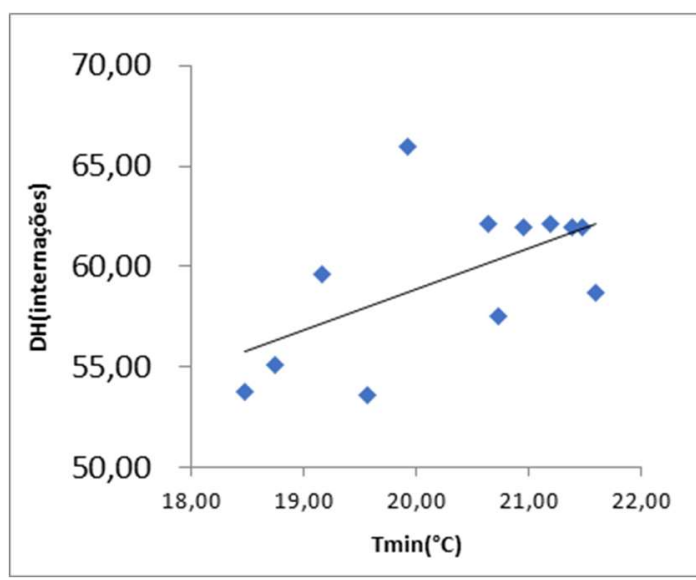

Figura 3: Diagrama de Dispersão Doenças Hipertensivas versus Tmin.

Por outro lado, verificou-se que as variáveis umidade relativa do ar, precipitação pluvial e pressão atmosférica apresentam uma correlação negativa indicando que à medida que seus valores aumentam, há uma tendência em diminuir os dos números das internações das DH. Nos estudos de Souza et al. (2011) observou-se resultado semelhante em relação a variável umidade relativa, visto que à medida que os valores de umidade relativa do ar baixam aumentam o número de casos de hipertensão arterial.

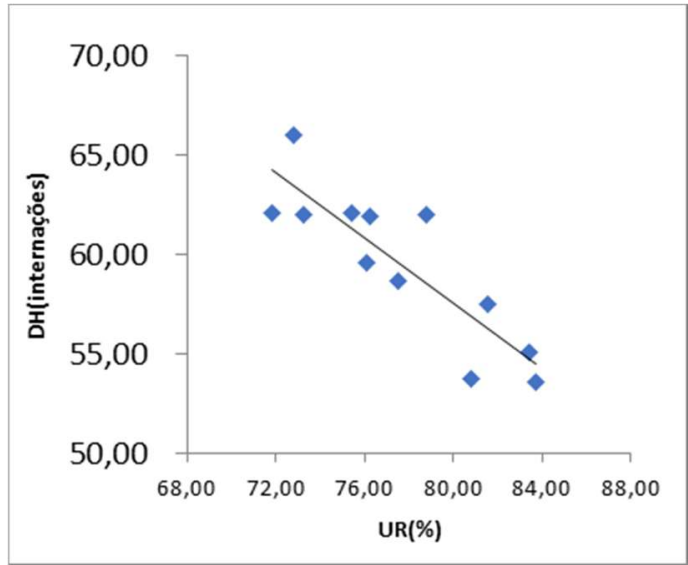

Figura 4: Diagrama de Dispersão Hipertensivas versus UR.

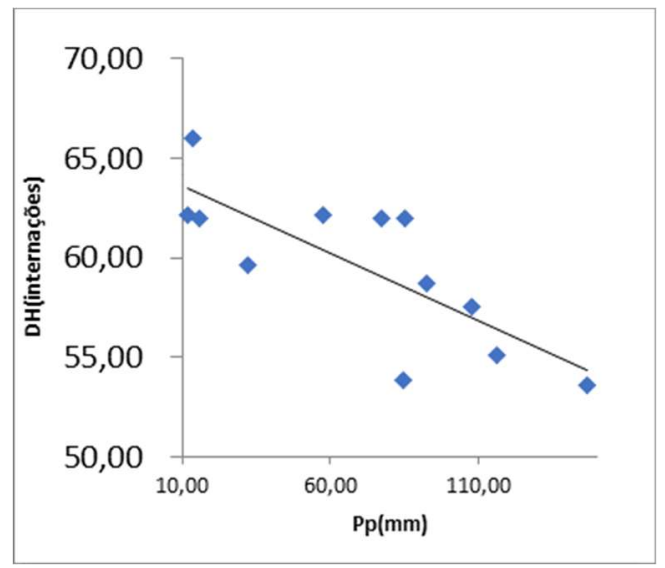

Figura 5: Diagrama de Dispersão Doenças Hipertensivas versus Pp.

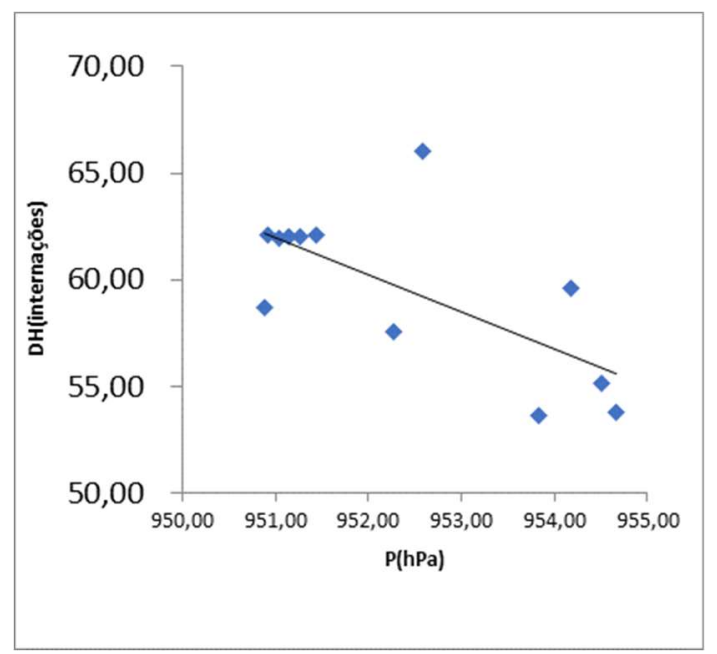

Figura 6: Diagrama de Dispersão Doenças Hipertensivas versus $P$.

\section{Correlações grupo das doenças isquêmicas do coração e as variáveis meteorológicas}


A Tabela 2 apresenta os resultados dos coeficientes de correlação Linear de Pearson $(r)$ referente à correlação das doenças isquêmicas do coração (DISC) versus as variáveis Meteorológicas.

Tabela 2: Matriz de Correlação DISC versus Variáveis Meteorológicas.

\begin{tabular}{lllllll}
\hline Ano & $\mathbf{T}_{\text {med }}$ & $\mathbf{T}_{\max }$ & $\mathbf{T}_{\min }$ & UR & Pp & $\mathbf{P}$ \\
\hline 1998 & 0,32 & 0,12 & 0,43 & 0,25 & 0,51 & $-0,25$ \\
1999 & 0,13 & 0,30 & 0,00 & $-0,64$ & $-0,38$ & $-0,07$ \\
2000 & 0,32 & 0,38 & 0,20 & $-0,43$ & $-0,37$ & $-0,25$ \\
2001 & $-0,22$ & $-0,17$ & 0,03 & $-0,12$ & 0,27 & $-0,14$ \\
2002 & 0,18 & 0,32 & 0,05 & $-0,30$ & $-0,10$ & $-0,05$ \\
2003 & 0,00 & 0,2 & $-0,16$ & $-0,42$ & $-0,48$ & 0,16 \\
2004 & 0,58 & 0,34 & 0,66 & $-0,27$ & 0,34 & $-0,65$ \\
2005 & 0,51 & 0,49 & 0,43 & $-0,20$ & $-0,50$ & $-0,42$ \\
2006 & 0,26 & 0,42 & 0,17 & $-0,50$ & $-0,47$ & $-0,07$ \\
2007 & 0,69 & 0,64 & 0,67 & $-0,32$ & $-0,22$ & $-0,68$ \\
2008 & 0,33 & 0,44 & 0,24 & $-0,72$ & $-0,40$ & $-0,13$ \\
2009 & 0,52 & 0,46 & 0,47 & $-0,34$ & $-0,44$ & $-0,49$ \\
2010 & 0,21 & 0,13 & 0,26 & 0,14 & 0,12 & $-0,02$ \\
2011 & 0,43 & 0,38 & 0,46 & $-0,22$ & $-0,26$ & $-0,50$ \\
2012 & 0,28 & 0,32 & 0,27 & $-0,31$ & $-0,28$ & $-0,14$ \\
2013 & $-0,16$ & $-0,25$ & $-0,12$ & 0,32 & 0,53 & 0,13 \\
\hline
\end{tabular}

De acordo com os resultados dos coeficientes de correlação Linear de Pearson (r), verificou-se que as temperaturas (média, máxima e mínima) apresentou no período de janeiro de 1998 a dezembro de 2013 uma predominância nos valores das correlações positivas, apresentando correlações medianas. Essa correlação positiva indica que à medida que os valores das temperaturas (média, máxima e mínima) aumentam, há uma tendência dos valores dos números das internações das doenças Isquêmicas do coração também crescer. Resultados semelhantes foram encontrados por Koken et al. (2003), os quais observaram em seu estudo que as temperaturas altas pareciam ser um importante fator para o aumento da frequência de internações por infarto no miocárdio que pertence ao grupo de doenças isquêmicas do coração. As Figuras 7, 8 e 9 ilustram as correlações lineares por meio dos diagramas de dispersões. Através de suas variáveis apresentam uma correlação linear quando os pontos do diagrama de dispersões se aproximam de uma reta.

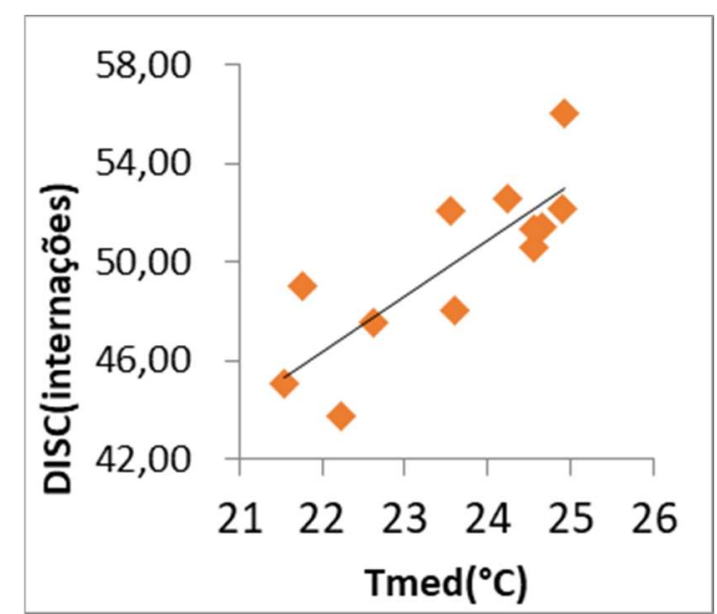

Figura 7: Diagrama de Dispersão DISC versus Tmed.

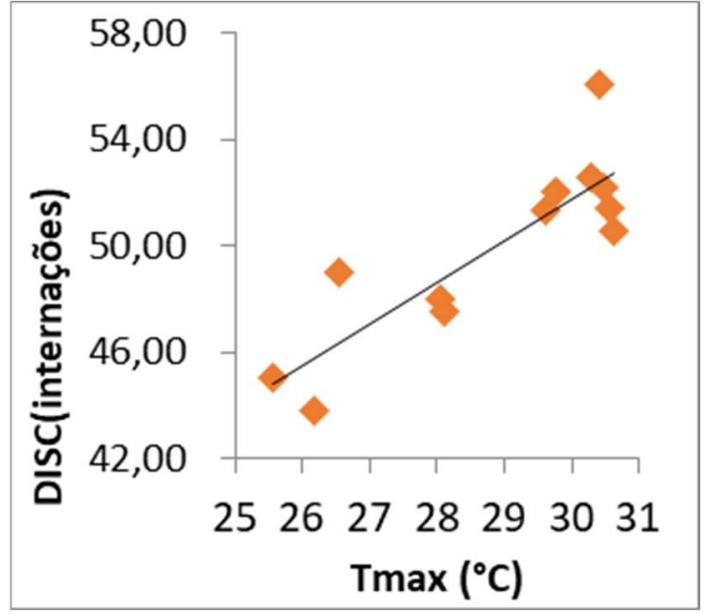

Figura 8: Diagrama de Dispersão DISC versus T Tmax. 


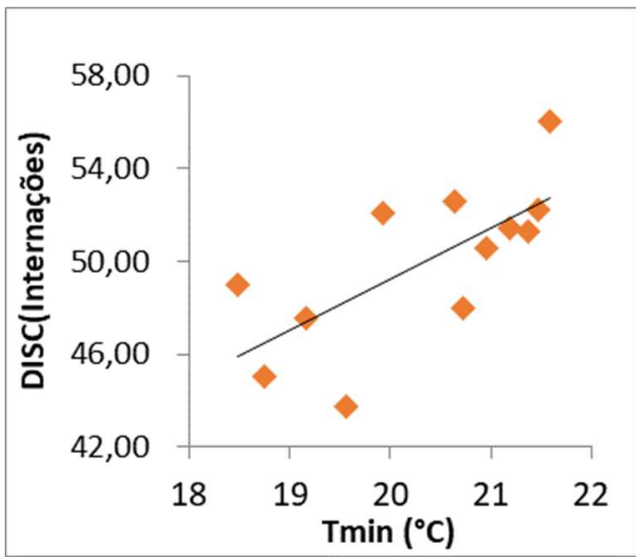

Figura 9: Diagrama de Dispersão DISC versus Tmin.

Por outro lado, verificou-se que as variáveis: umidade relativa do ar, precipitação pluvial e pressão apresentam uma correlação negativa indicando que à medida que os valores da umidade relativa do ar, precipitação pluvial e pressão aumentam, há uma tendência em diminuir os valores nos números das internações das Doenças Isquêmicas (DISQ). As Figuras 10, 11 e 12 ilustram as correlações lineares através dos diagramas de dispersões.

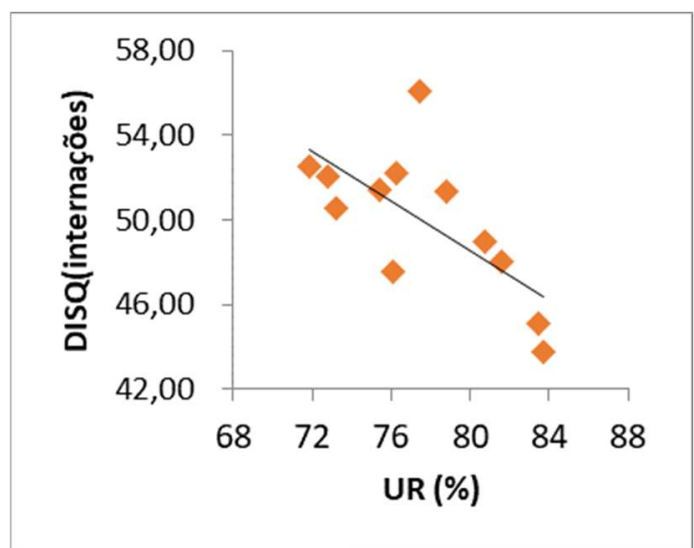

Figura 10: Diagrama de Dispersão DISC versus UR.

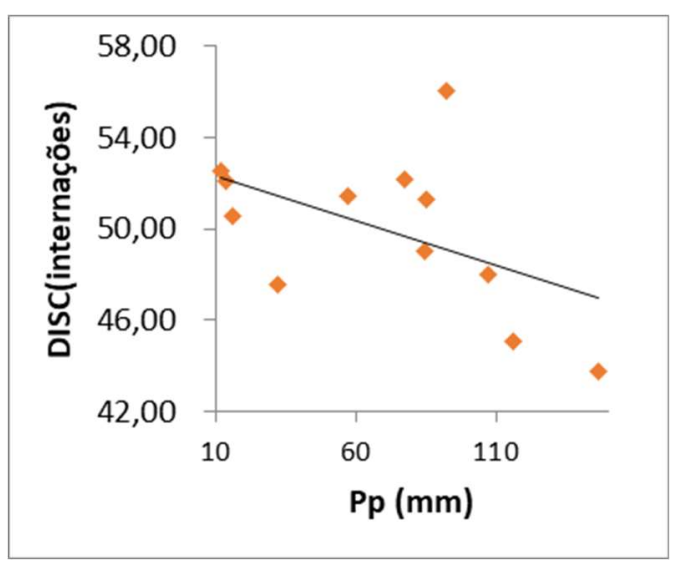

Figura 11: Diagrama de Dispersão DISC versus Pp.

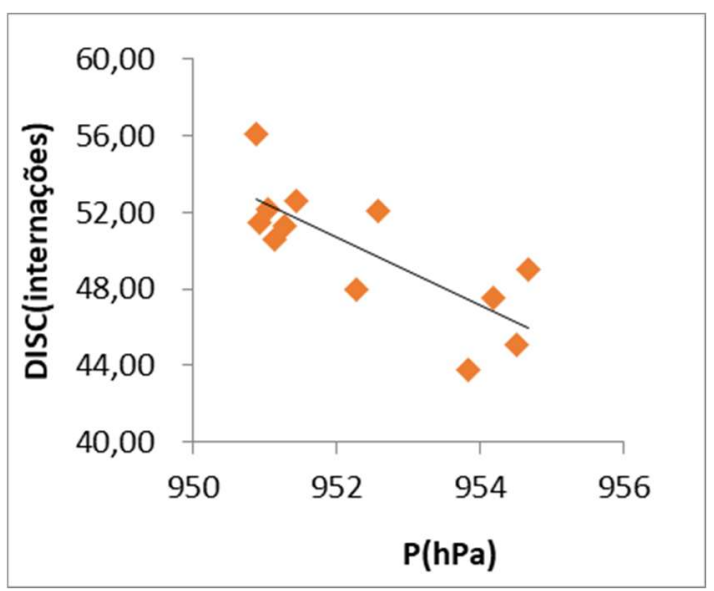

Figura 12: Diagrama de Dispersão DISC versus $P$.

\section{Correlações insuficiência cardíaca e as variáveis meteorológicas}

A Tabela 3 apresenta os resultados dos coeficientes de correlação Linear de Pearson $(r)$ referente à correlação das doenças Insuficiência Cardíaca (IC) versus as variáveis Meteorológicas. 
Tabela 3: Matriz de Correlação IC versus Variáveis Meteorológicas.

\begin{tabular}{lllllll} 
Ano & $\mathbf{T}_{\text {med }}$ & $\mathbf{T}_{\max }$ & $\mathbf{T}_{\min }$ & UR & Pp & P \\
\hline 1998 & $-0,19$ & $-0,32$ & $-0,16$ & 0,29 & 0,42 & 0,40 \\
1999 & 0,18 & 0,14 & 0,20 & 0,11 & 0,25 & $-0,28$ \\
2000 & 0,07 & 0,07 & $-0,30$ & $-0,26$ & $-0,39$ & 0,19 \\
2001 & $-0,45$ & $-0,52$ & $-0,30$ & 0,32 & 0,48 & 0,15 \\
2002 & 0,12 & 0,32 & 0,00 & $-0,40$ & $-0,06$ & $-0,08$ \\
2003 & 0,18 & 0,33 & 0,02 & $-0,45$ & $-0,49$ & $-0,14$ \\
2004 & 0,49 & 0,29 & 0,61 & $-0,13$ & 0,27 & $-0,44$ \\
2005 & $-0,05$ & $-0,28$ & 0,16 & 0,63 & 0,57 & 0,08 \\
2006 & 0,16 & 0,12 & 0,15 & $-0,08$ & 0,06 & $-0,12$ \\
2007 & 0,67 & 0,73 & 0,61 & $-0,56$ & $-0,44$ & $-0,76$ \\
2008 & 0,53 & 0,54 & 0,52 & $-0,17$ & 0,13 & $-0,50$ \\
2009 & 0,60 & 0,5 & 0,59 & $-0,25$ & $-0,30$ & $-0,50$ \\
2010 & $-0,01$ & $-0,03$ & $-0,03$ & 0,01 & $-0,26$ & $-0,01$ \\
2011 & $-0,47$ & $-0,32$ & $-0,62$ & 0,06 & $-0,27$ & 0,73 \\
2012 & $-0,22$ & $-0,06$ & $-0,38$ & $-0,29$ & $-0,53$ & 0,28 \\
2013 & 0,30 & 0,41 & 0,24 & $-0,56$ & $-0,51$ & $-0,29$ \\
\hline
\end{tabular}

De acordo com os resultados dos coeficientes de correlação Linear de Pearson (r), verificou-se que as temperaturas (média, máxima e mínima) apresentou no período de janeiro de 1998 a dezembro de 2013 uma predominância em valores das correlações positivas, apresentando correlações medianas. Essa correlação positiva indica os valores das temperaturas (média, máxima e mínima) apresentam um leve aumento nos registros de casos de Insuficiência Cardíaca (IC). Conforme pesquisa realizada por Koken et al. (2003), constatou-se que o aumento das temperaturas também estava relacionado ao aumento na frequência de internações por insuficiência cardíaca. As Figuras 13, 14 e 15 ilustram as correlações lineares através dos diagramas de dispersões.

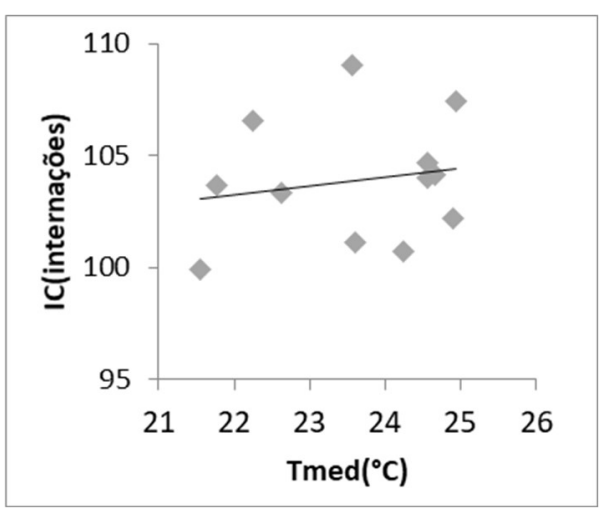

Figura 13: Diagrama de Dispersão IC versus Tmax.

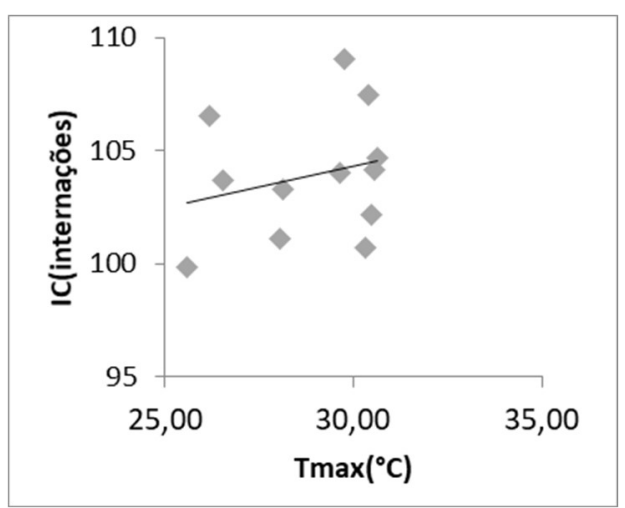

Figura 14: Diagrama de Dispersão IC versus Tmed.

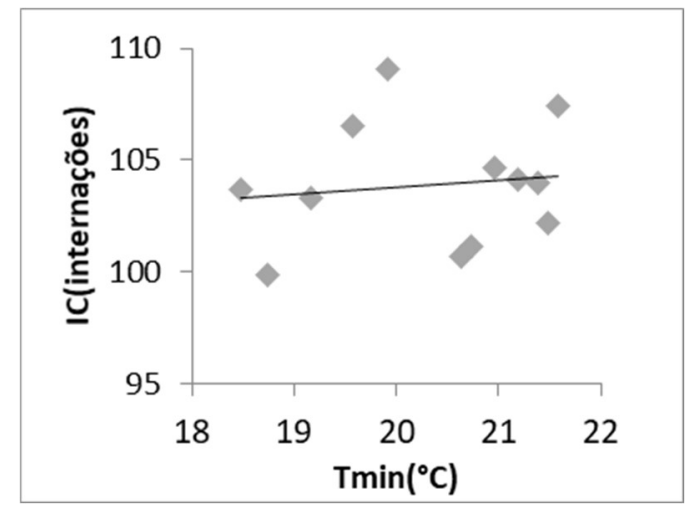

Figura 15: Diagrama de Dispersão IC versus Tmin

Verificou-se que as variáveis: umidade relativa do ar, precipitação pluvial e pressão apresentam uma 
correlação negativa indicando que à medida que os valores da umidade relativa do ar, precipitação pluvial e pressão aumentam, há uma tendência em diminuir os valores nos números das internações da Insuficiência Cardíaca (IC). As Figuras 16, 17 e 18 ilustram as correlações lineares através dos diagramas de dispersões.

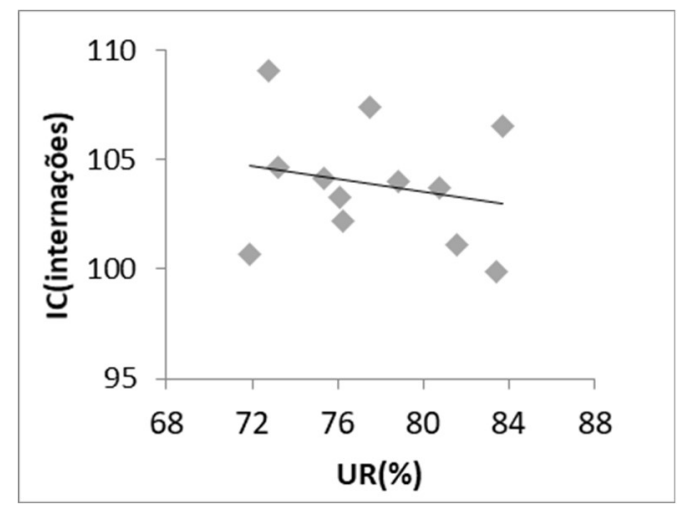

Figura 16: Diagrama de Dispersão IC versus UR.

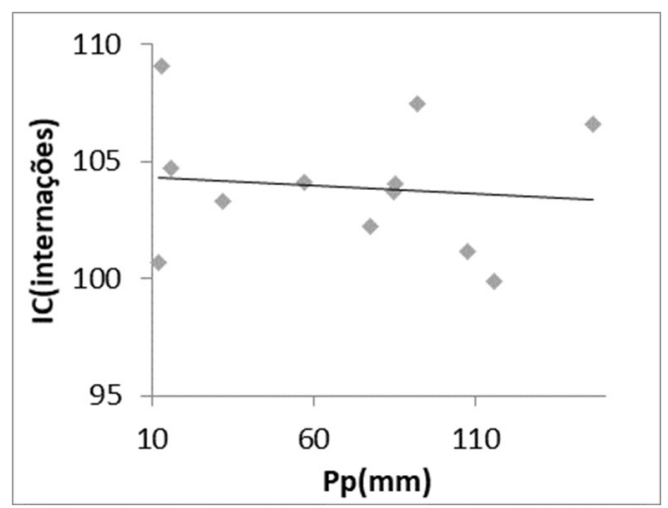

Figura 17: Diagrama de Dispersão IC versus Pp.

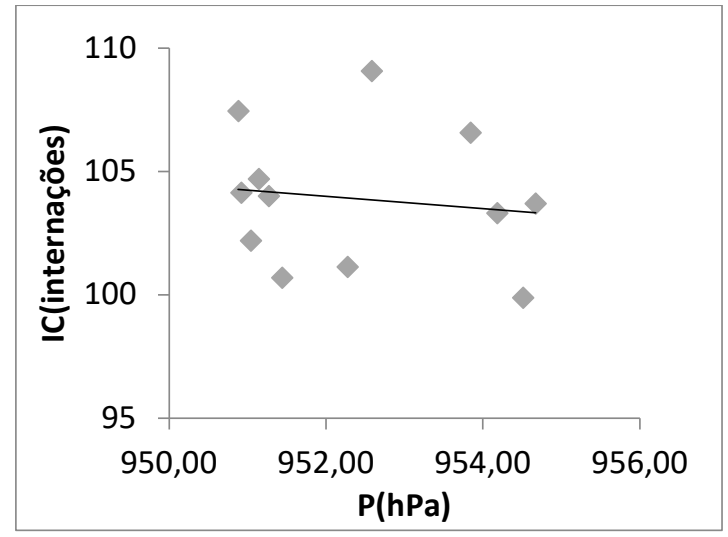

Figura 18: Diagrama de Dispersão IC versus $P$.

Através dos valores correlações de Pearson que as variáveis meteorológicas apresentam associação com as doenças do aparelho circulatório ao longo do período de janeiro de 1998 a dezembro de 2013, indicando que existe correlação mediana quando relacionada cada variável metrológicas com cada doença.

Com a finalidade de determinar a influência das variáveis independentes (variáveis metrológicas) atuando em conjunto, foi realizada a análise de regressão linear múltipla para verificar como melhor explicam o comportamento da variável Dependente (doenças do aparelho circulatório).

\section{Regressão Linear Múltipla (RLM)}

De modo a avaliar a influência das variáveis meteorológicas na explicação do comportamento dos grupos das doenças do aparelho circulatório foi aplicada a análise estatística por RLM.

O objetivo da análise de Regressão Linear Múltipla é determinar a força de cada uma das variáveis independentes que, em conjunto, melhor explicam o comportamento da variável dependente. No nosso estudo prevemos as mudanças das variáveis dependentes: Doenças Hipertensivas (DH), Doenças Isquêmicas do coração (DISC) e Insuficiência Cardíaca (IC)

No estudo foram obtidos modelos matemáticos através da análise estatística da Regressão Linear múltipla, para conhecer quanto e se as variáveis meteorológicas: temperatura (média, máxima e mínima), 
umidade relativa do ar, precipitação pluvial e pressão atmosférica influenciam no número de internações dos grupos de doenças do aparelho circulatório Doenças Hipertensivas (DH), Doenças Isquêmicas do coração (DISC) e Insuficiência Cardíaca (IC).

\section{Influência das variáveis meteorológicas nas doenças hipertensivas (DH)}

Os resultados dos testes da análise de Regressão Linear Múltipla apresentada na Tabela 4, mostram o quanto as variáveis meteorológicas explicam o comportamento do grupo das Doenças Hipertensivas. Estes resultados indicam que as variáveis analisadas juntas explicam $43 \%\left(R^{2}\right.$ adj $\left.=0,43\right)$, do comportamento total mensal das internações das Doenças Hipertensivas, explicado assim através do modelo matemático obtido pela equação 1 .

Tabela 4: Sumário do Modelo Regressão múltipla (Variável dependente: DH).

\begin{tabular}{llllllll}
\hline Estimados & Coeficientes & Erro padrão & Stat $t$ & valor-p & $R$ & $R^{2}$ & $R^{2}$ adj \\
\hline Intercepto & $6.422,64$ & 1619,78 & 3,97 & 0,00 & $67 \%$ & $45 \%$ & $43 \%$ \\
$T_{\operatorname{med}}$ & 37,18 & 5,32 & 6,99 & 0,00 & & & \\
$T_{\max }$ & $-6,67$ & 2,34 & $-7,11$ & 0,00 & & \\
$T_{\min }$ & $-26,75$ & 4,00 & $-6,69$ & 0,00 & & \\
UR & $-3,03$ & 0,49 & $-6,22$ & 0,00 & & \\
$P p$ & 0,03 & 2,32 & 0,02 & & \\
$P$ & $-6,29$ & 1,67 & $-3,77$ & 0,00 & & \\
\hline
\end{tabular}

Conforme os resultados da coluna dos coeficientes da Tabela 4, permitiu-se escrever a equação 1 que representa o modelo matemático empírico das Doenças Hipertensivas (DH) estabelecido pelas as variáveis independentes, onde os coeficientes em negrito são os efeitos estatisticamente significativos ao nível de $95 \%$ de confiança, pois os valores-p são inferiores a 0,05, podendo assegurar que o modelo da regressão (Equação 1) é útil para predizer os valores dos números das internações das Doenças Hipertensivas $(\mathrm{DH})$.

$\mathrm{DH}=6422,64+37,18 \mathrm{~T}_{\text {med }}-6,67 \mathrm{~T}_{\max }-26,75 \mathrm{~T}_{\min }-3,03 \mathrm{UR}+0,08 \mathrm{Pp}-6,29 \mathrm{P}$ Equação (1)

\section{Influência das variáveis meteorológicas nas doenças isquêmicas docoração (DISC)}

Os resultados dos testes da análise de Regressão Linear Múltipla apresentada na Tabela 5, mostram o quanto as variáveis meteorológicas explicam o comportamento das Doenças Isquêmicas do Coração. Estes resultados indicam que as variáveis analisadas juntas explicam $36 \%\left(R^{2}\right.$ adj $\left.=0,36\right)$, do comportamento total mensal das internações nas Doenças Isquêmicas do Coração (DISC), explicado assim através do modelo matemático obtido pela equação 2 .

Tabela 5: Sumário do Modelo Regressão múltipla (Variável dependente: DISC).

\begin{tabular}{lcccccc}
\hline Estimados & Coeficientes & Erro padrão & Stat $t$ & valor-p & $R$ & $R^{2}$ \\
\hline Intercepto & $2.083,07$ & $1.027,21$ & 2,03 & 0,04 & $62 \%$ & $38 \%$ \\
$T_{\operatorname{med}}$ & 20,91 & 3,37 & 6,20 & 0,00 & $36 \%$ \\
$T_{\max }$ & $-9,85$ & 1,49 & $-6,63$ & 0,00 & \\
$T_{\min }$ & $-12,04$ & 2,54 & $-4,75$ & 0,00 & \\
UR & $-1,90$ & 0,31 & $-6,14$ & 0,00 & \\
Pp & 0,03 & 0,02 & 1,54 & 0,13 & \\
$P$ & $-1,95$ & 1,06 & $-1,84$ & 0,07 & \\
\hline
\end{tabular}


A Tabela 5 apresenta os resultados dos coeficientes que permite escrever a equação 2 que representa o modelo matemático empírico das doenças Isquêmicas do coração (DISC) estabelecido pelas variáveis independentes, onde os coeficientes em negrito são os efeitos estatisticamente significativos ao nível de 95\% de confiança, pois os valores-p são inferiores a 0,05, podendo assegurar que o modelo da regressão (Equação 2) é útil para predizer os valores dos números das internações por doenças isquêmicas do coração (DISC).

DISC $=2083,07+20,91 T_{\operatorname{med}}-9,85 T_{\max }-12,04 T_{\min }-1,90 U R+0,03 P p-1,95 P$ Equação (2)

\section{Influência das variáveis meteorológicas na insuficiência cardíaca}

Os resultados dos testes da análise de Regressão Linear Múltipla apresentada na Tabela 6 mostram o quanto as variáveis meteorológicas explicam o comportamento da Insuficiência Cardíaca. Estes resultados indicam que as variáveis analisadas juntas explicam $34 \%\left(R^{2}\right.$ adj $\left.=0,34\right)$, do comportamento total mensal das internações da Insuficiência Cardíaca (IC), explicando assim através do modelo matemático obtido pela equação 3.

Tabela 6: Sumário do Modelo Regressão múltipla (Variável dependente: IC).

\begin{tabular}{lcclllll}
\hline Estimados & Coeficientes & Erro padrão & Stat $t$ & valor-p & $R$ & $R^{2}$ & $R^{2}$ adj \\
\hline Intercepto & $9.173,89$ & $2.299,20$ & 3,99 & 0,00 & $60 \%$ & $36 \%$ & $34 \%$ \\
$T_{\operatorname{med}}$ & 42,81 & 7,55 & 5,67 & 0,00 & & & \\
$T_{\max }$ & $-19,58$ & 3,33 & $-5,89$ & 0,00 & & \\
$T_{\min }$ & $-34,05$ & 5,68 & $-6,00$ & 0,00 & & \\
$U R$ & $-3,24$ & 0,69 & $-4,69$ & 0,00 & & \\
$P p$ & 0,06 & 0,05 & 1,26 & 0,21 & & \\
$P$ & $-9,00$ & 2,37 & $-3,80$ & 0,00 & & \\
\hline
\end{tabular}

Conforme os resultados da coluna dos coeficientes da Tabela 6, permitiu-se escrever a equação 3 que representa o modelo matemático empírico da Insuficiência Cardíaca (IC) estabelecido pelas as variáveis independentes, onde os coeficientes em negrito são os efeitos estatisticamente significativos ao nível de 95\% de confiança, pois os valores-p são inferiores a 0,05, podendo assegurar que o modelo da regressão (Equação 3) é útil para predizer os valores dos números das internações da Insuficiência Cardíaca (IC).

$$
I C=9173,89+42,81 T_{\operatorname{med}}-19,58 T_{\max }-34,05 T_{\min }-3,24 U R+0,06 \mathrm{Pp}-9 \text { P Equação (3) }
$$

\section{Performance dos Modelos Matemáticos}

Para que uma regressão não seja apenas estatisticamente significativa, mas também útil para fins preditivos, o valor da razão entre o $\mathrm{F}_{\text {calculado }}$ e o $\mathrm{F}_{\text {tabelado }}$ deve ser no mínimo maior que quatro (BARROS NETO et al., 1996). Tais resultados foram obtidos na análise de Regressão Linear Múltipla das doenças: Hipertensão Arterial, Isquemia e Insuficiência Cardíaca.

As Figuras 19, 20 e 21 apresentam os gráficos da performance dos modelos matemáticos obtidos para o número de internações das doenças do aparelho circulatório (doenças hipertensivas, doenças Isquêmicas do coração e Insuficiência Cardíaca) através dos valores preditos quando comparado com os valores observados.

Verifica-se para os três tipos de doenças, que as performances do modelo obtido através dos valores 
preditos são bem semelhantes com a performance dos valores observados.

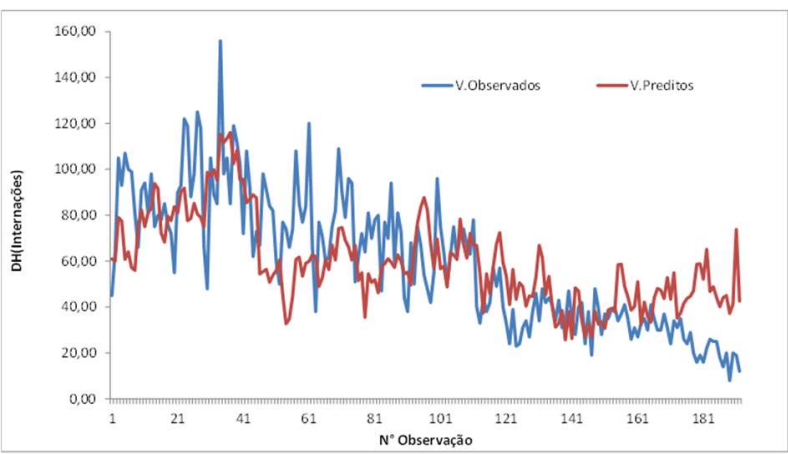

Figura 19: Performance do Modelo Matemático Doenças hipertensivas (DH).

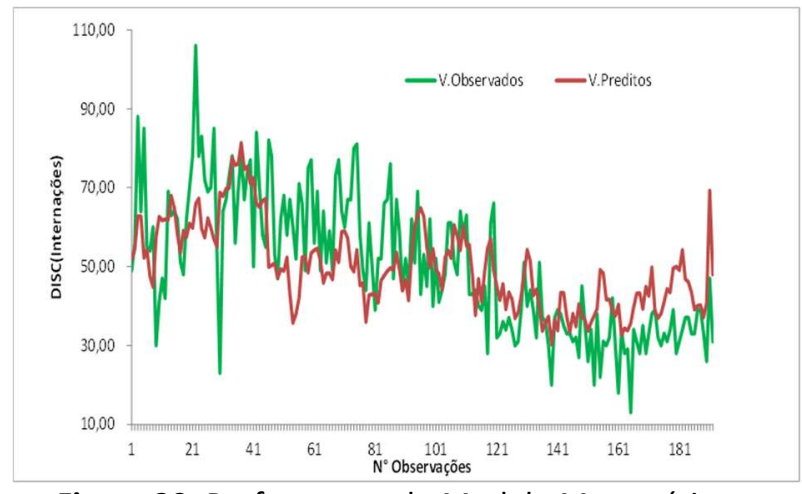

Figura 20: Performance do Modelo Matemático Doenças isquêmicas do coração (DISC).

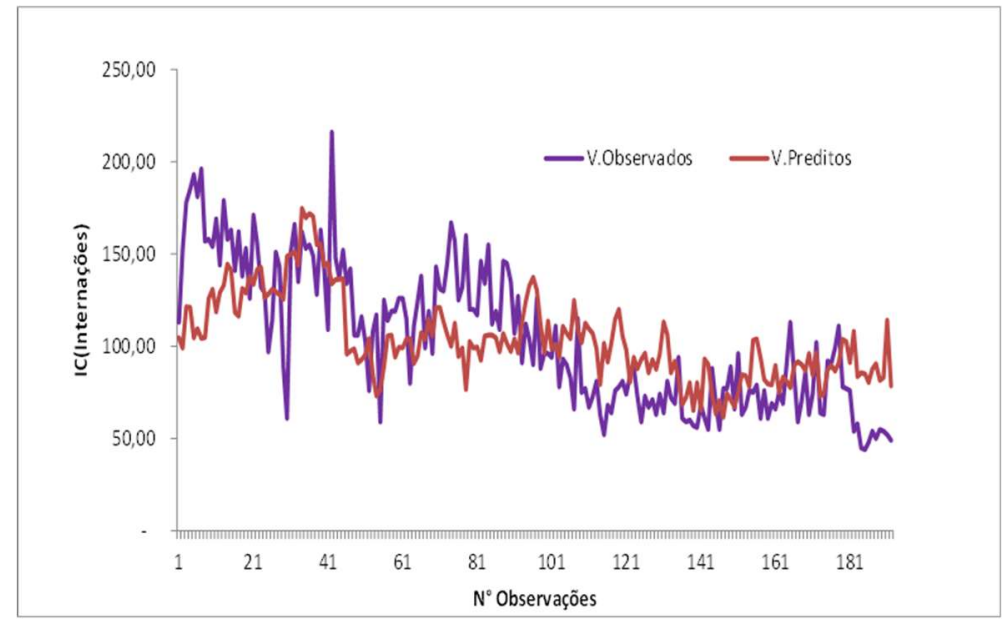

Figura 21: Performance do Modelo Matemático - Insuficiência Cardíaca (IC).

\section{CONCLUSÕES}

O clima entre outros fatores pode ser um elemento desencadeador de morbidades através de seus atributos (temperatura do ar, umidade relativa do ar, precipitação pluviométrica e pressão atmosférica) que interfere no bem-estar das pessoas. No entanto, não se pode colocar o clima como o único responsável pelo desencadeamento de enfermidades. Tal situação quando somada a outros fatores de risco tais como, estilo de vida, hábitos alimentares, doenças pré-existentes podem contribuir para o agravamento das mesmas e favorecer o aparecimento de outras.

Com base nos resultados obtidos através das análises estatísticas, pode-se concluir que: 1) Esse estudo encontrou correlação entre os elementos climáticos e internações por doenças do aparelho circulatório. A correlação verificada com os elementos climáticos indica que as internações por Doenças do aparelho circulatório apresentam correlação de média a forte. Sendo as variáveis temperatura média e umidade relativa do ar as que mais influenciam no comportamento destas doenças; 2) Os elementos climáticos juntos explicam: 43\% das internações por doenças hipertensivas, 36\% das internações por doenças isquêmicas do coração e 34 \% das internações por insuficiência cardíaca; 3) Os modelos matemáticos obtidos através da regressão linear múltipla são úteis para fins preditivos, devido o valor da razão entre o $F_{\text {calculado }} \mathrm{e}$ o $F_{\text {tabelado }}$ ser maior que 4 ; 4) A performance dos valores preditos apresenta comportamento semelhante aos 
valores observados.

\section{REFERÊNCIAS}

BARROS NETO, B.; SCARMINIO, I. S.; BRUNS, R. E.. Planejamento e otimização de experimentos. 2 ed. Campinas: UNICAMP, 1996.

GONÇALVES, F. L. T.; BRAUN, S.; DIAS, P. L. S.; SHAROVSKY, R.. Influences of the weather and air pollutants on cardiovascular disease in the metropolitan area of São Paulo. Environmental Research, v.104, p.275-281, 2007.

KEATINGE, W. R.; COLESHAW, S. R. K.; COTTER, F.; MATTOCK, M.; MURPHY, M.; CHELLIAH, R.. Increases in platelet and red cell counts, blood viscosity, and arterial pressure during mild surface cooling: factors in mortality from coronary and cerebral thrombosis in winter. British Medical Journal, v.289, 1984.

KOKEN, P. J. M.; PIVER, W. T.; YE, F.; ELIXHAUSER, A.; OLSEN, L. M.; PORTIER, C. J.. Temperature, air pollution, and hospitalization for cardiovascular diseases among elderly people in Denver. Environmental Health Perspectives, v.111, n.10, p.1312-1317, 2003.

MURARA, P. G. S.. Variabilidade climática e doenças circulatórias e respiratórias em Florianópolis (SC): uma contribuição à climatologia médica. Dissertação (Mestrado em Geografia) - Universidade Federal de Santa Catarina, Florianópolis, 2012.

SCHWARTZ, J.; SAMET, J. M.; PATZ, J. A.. Hospital admissions for heart disease the effects of temperature and humidity. Epidemiology, v.15, n.6, p.755-761, 2004.

SERRANO JUNIOR, C. V.; TIMERMAN, A.; STEFANINI, E. Tratado de cardiologia SOCESP. 2 ed. São Paulo: Manole, 2009.

SILVA, P. M. F.; PEREIRA, H. S.; DANTAS, R. T.. Infarto agudo do miocárdio: influência e correlação de variáveis meteorológicas. In: Saúde ambiental: um olhar reflexivo. Florianópolis: EPGRAF, 2013.

SOUZA, V. C.; SOUZA, E. P.; SILVA, S. S. F.. Hipertensão arterial - uma visão sazonal do Programa Saúde da Família no Município de Areia - PB. Revista de Saúde Pública de Santa Catarina, v.4, p.8-20, 2011.

PITTON, S. E. C.; DOMINGOS, A. E.. Tempo e doenças: efeitos dos parâmetros climáticos nas crises hipertensivas nos moradores de Santa Gertrudes- SP. Estudos Geográficos, Rio Claro, v.2, n.1, 2004.

A CBPC - Companhia Brasileira de Produção Científica (CNPJ: 11.221.422/0001-03) detém os direitos materiais desta publicação. Os direitos referem-se à publicação do trabalho em qualquer parte do mundo, incluindo os direitos às renovações, expansões e disseminaçães da contribuição, bem como outros direitos subsidiários. Todos os trabalhos publicados eletronicamente poderão posteriormente ser publicados em coletâneas impressas sob coordenação da Sustenere Publishing, da Companhia Brasileira de Produção Científica e seus parceiros autorizados. Os (as) autores (as) preservam os direitos autorais, mas não têm permissão para a publicação da contribuição em outro meio, impresso ou digital, em português ou em tradução. 